A. Terras.

Nagoya Math. J

Vol. 44 (1971), 89-95

\title{
FUNCTIONAL EQUATIONS OF GENERALIZED EPSTEIN ZETA FUNCTIONS IN SEVERAL COMPLEX VARIABLES
}

\author{
AUDREY A. TERRAS
}

Let $S^{(n)}$ be the matrix of a positive definite quadratic form and $\left(\rho_{1}, \cdots, \rho_{r-1}\right) \in \boldsymbol{C}^{r-1}$. Define

$$
\zeta_{n_{1}}, \cdots, n_{r}\left(S, \rho_{1}, \cdots, \rho_{r-1}\right)=\sum_{i=1}^{r-1}\left|S\left[U_{i}\right]\right|^{-\rho_{i}} .
$$

Here the sum is over unimodular matrices $U^{(n)}=\left(U_{i}^{\left.\left(n, N_{i}\right) *\right)}\right.$ which lie in a complete set of representatives for the equivalence relation $U \sim V$ if $U=V P$, with $P$ unimodular and having block form

$$
P=\left(\begin{array}{ccc}
P_{!}^{\left(n_{1}\right)} & & * \\
& * & \\
0 & P_{r}^{\left(n_{r}\right)}
\end{array}\right) .
$$

The following notation shall be used throughout:

$$
\begin{aligned}
& A^{(n, m)} \text { for an } n \text { by } m \text { matrix } A \\
& A^{(n)}=A^{(n, n)} \\
& |A|=\text { determinant of } A \\
& S[A]={ }^{t} A S A,{ }^{t} A \text { being the transpose of } A \\
& N_{i}=\sum_{j=1}^{i} n_{j}, \quad i=1,2, \cdots, \gamma, N_{r}=n=\sum_{j=1}^{r} n_{j} .
\end{aligned}
$$

$A$ unimodular matrix $U^{(n)}$ is one with integral entries and determinant \pm 1 .

The function (1) is clearly a generalization of Epstein's zeta function, as well as a generalization of functions defined by Koecher [1], Maass [3], and Selberg [4], [5], [6]. It can also be viewed as an Eisenstein series; for which, see Langlands [2].

If $n_{i}=1$ for all $i=1,2, \cdots, \gamma$ and $\gamma=n$, denote the function defined by $(1)$ as $\zeta_{(n)}=\zeta_{1}, \cdots, 1$.

Received January 29, 1971. 
We know from [7] that (1) converges for $\operatorname{Re} \rho_{i}>\frac{1}{2} n$, for $i=1,2, \cdots$, $r-1$. It was also shown in [7] that

$$
\zeta_{n_{1}}, \cdots, n_{r}\left(S, \rho_{N_{1}}, \cdots, \rho_{N_{r-1}}\right)=\left.\zeta_{(n)}(S, \rho)\right|_{\rho_{i}=0 \text {, for } i \notin\left\{N_{1}, \cdots, N_{r}\right\} .}
$$

Also from [7], every permutation $\sigma$ of $\{1,2, \cdots, n]$ induces a functional equation of $\zeta_{(n)}(S, \rho)$. More precisely, one introduces the new variables $z_{i}, i=1,2, \cdots, n$ with $\rho_{i}=z_{i+1}-z_{i}+\frac{1}{2}$. That means

$$
\rho=\left(\begin{array}{c}
\rho_{1} \\
\vdots \\
\vdots \\
\rho_{n}
\end{array}\right)=\left(\begin{array}{rrr}
-1+1 & & \\
-1+1 & 0 \\
& \cdot & \\
0 & -{ }_{-1}
\end{array}\right)\left(\begin{array}{c}
z_{1} \\
\vdots \\
\vdots \\
z_{n}
\end{array}\right)+\left(\begin{array}{c}
\frac{1}{2} \\
\vdots \\
\frac{1}{2}
\end{array}\right)=A z+\frac{1}{2} .
$$

Define $\phi(x)=\Gamma(x) \zeta(2 x)$. Here $\zeta$ denotes the ordinary Riemann zeta function. Now define

$$
\Phi^{\prime}(z)=|S|^{z_{n}-\frac{1}{2}} \pi^{-2} \sum_{j=1}^{n} j z_{j} \prod_{1 \leq i<j \leq n} \phi\left(z_{j}-z_{i}+\frac{1}{2}\right) .
$$

Then the functional equations of $\zeta_{(n)}(S, \rho)=\zeta_{(n)}^{\prime}(S, z)$, for $\rho=A z+\frac{1}{2}$, are

$$
\Phi^{\prime}\left(z^{\sigma}\right) \zeta_{(n)}^{\prime}\left(S, z^{\sigma}\right)=\Phi^{\prime}(z) \zeta_{(n)}^{\prime}(S, z),
$$

where $\left(z^{\sigma}\right)_{i}=z_{\sigma(i)}$, that is,

$$
z^{o}=P^{o} z, \text { where } P^{o}=\left(\delta_{o(i) j}\right), \delta_{i j} \text { being }
$$

the usual Kronecker delta.

One can rewrite the functional equations in terms of the variables $\rho$ by defining

$$
\begin{aligned}
& \Phi(\rho)=\Phi^{\prime}(z), \text { for } \rho=A z+\frac{1}{2} ; \\
& \rho^{\sigma}=A P^{o} A^{-1}\left(\rho-\frac{1}{2}\right)+\frac{1}{2} ; \\
& F(\rho, \sigma)=\frac{\Phi(\rho)}{\Phi\left(\rho^{\sigma}\right)} .
\end{aligned}
$$

Then the functional equations become

$$
\zeta_{(n)}\left(S, \rho^{\sigma}\right)=F(\rho, \sigma) \zeta_{(n)}(S, \rho) .
$$

Note that we have defined the map $\rho \rightarrow \rho^{\circ}$ to be the map induced on the 
$\rho$ coordinates by the map $z \rightarrow z^{\sigma}$. It is clear that $\left(\rho^{\sigma}\right)_{i} \in Q\left[\rho_{1}, \cdots, \rho_{n-1}\right]$, for $i=1, \cdots, n-1$.

Now equation (2) suggests that some of the equations (3) induce functional equations or relations on the functions $\zeta_{n_{1}}, \cdots, n_{r}$. The purpose now is to discover which $r$-tuples $\left(n_{1}, \cdots, n_{r}\right),\left(m_{1}, \cdots, m_{r}\right)$ such that $\sum_{i=1}^{r} n_{i}=n$ $=\sum_{i=1}^{r} m_{i}$ have the property that the corresponding zeta functions $\zeta_{n_{1}}, \cdots, n_{r}$ and $\zeta_{m_{1}}, \cdots, m_{r}$ are related by (3) for some $\sigma$. A result in this direction is proved in [7] for $\gamma=2$. Relations were obtained between $\zeta_{i, n-i}$ and $\zeta_{n-i, i}$ in that case for $i=1,2, \cdots, n-1$. This means that if $i=n-i$, a nontrivial functional equation is induced on $\zeta_{i, i}$ by some permutation of $\{1,2$, $\cdots, 2 i\}$, in (3).

In order to state the problem more precisely, define $F_{n_{1}, \cdots, n_{r}}(\rho, \sigma)=$

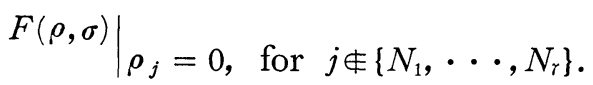

We say that for $\left(n_{1}, \cdots, n_{r}\right)$ and $\left(m_{1}, \cdots, m_{r}\right)$ such that $\sum_{i=1}^{r} n_{i}=n=\sum_{i=1}^{r} m_{i}$,

$$
\zeta_{n_{1}}, \cdots, n_{\gamma} \sim \zeta_{m_{1}}, \cdots, m_{\gamma}
$$

if and only if there exists a permutation $\sigma$ of $\{1,2, \cdots, n\}$ such that setting $\rho_{j}=0$, for $j \notin\left\{N_{1}, \cdots, N_{r}\right\}$ in (3), one obtains:

$$
\begin{array}{r}
F_{n_{1}, \cdots, n_{\gamma}}(\rho, \sigma) \zeta_{n_{1}, \cdots, n_{\gamma}}\left(S, \rho_{\left.N_{1}, \cdots, \rho_{N_{r-1}}\right)}\right. \\
=\zeta_{m_{1}, \cdots, m_{r}}\left(S, \rho_{M_{1}}^{\prime}, \cdots, \rho_{M_{r-1}}^{\prime}\right) .
\end{array}
$$

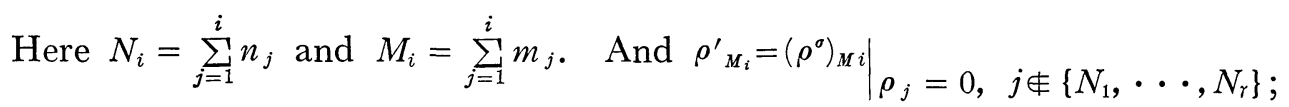
So $\rho_{M_{i}}^{\prime} \in \boldsymbol{Q}\left[\rho_{N_{1}}, \cdots, \rho_{N_{\gamma-1}}\right]$.

We shall soon see that " $\sim$ " is indeed an equivalence relation.

Clearly $\zeta_{n_{1}, \cdots, n_{\gamma}} \sim \zeta_{m_{1}}, \cdots, m_{\gamma}$ under $\sigma$ is equivalent to the condition that the $\rho$ 's which are set equal to zero to produce $\zeta_{n_{1}}, \cdots, n_{r}$ from $\zeta_{(n)}$ must be sent by the map $\rho \rightarrow \rho^{\sigma}$ to the $\rho$ 's which are set equal to zero to produce $\zeta_{m_{1}}, \cdots, m_{r}$ from $\zeta_{(n)}$.

Define $\mathscr{H}_{n_{1}, \cdots, n_{\gamma}}$ to be the set of $n-r$ hyperplanes defined by $z_{j+1}-z_{j}$ $+\frac{1}{2}=0$, for $j \notin\left\{N_{1}, \cdots, N_{r}\right\}$. Recall that $N_{i}=\sum_{j=1}^{i} n_{j}, i=1,2, \cdots, \gamma$. It is obvious that $\zeta_{n_{1}, \cdots, n_{r}} \sim \zeta_{m_{1}}, \cdots, m_{r}$ under $\sigma$ is equivalent to requiring that the map $z \rightarrow z^{\sigma}$ take the set $\mathscr{H}_{n_{1}, \cdots, n_{r}}$ one-to-one, onto the set $\mathscr{H}_{m_{1}, \cdots, m_{r}}$. 
THEOREM 1. For every $\left(n_{1}, \cdots, n_{r}\right)$ and $\left(m_{1}, \cdots, m_{r}\right)$ such that $\sum_{i=1}^{r} n_{i}=n=\sum_{i=1}^{r} m_{i}$.

$$
\zeta_{n_{1}, \cdots, n_{r}} \sim \zeta_{m_{1}, \cdots, m_{r}}
$$

if and only if there exists a permutation $\mu$ of $\{1,2, \cdots, \gamma\}$ such that $n_{i}=m_{\mu(i)}$.

Proof. Suppose for some permutation $\mu$ of $\{1,2, \cdots, \gamma\}, n_{i}=m_{\mu(i)}$. Define a permutation $\sigma_{\mu}$ of $\{1,2, \cdots, n\}$ as follows. Set $N_{i}=\sum_{j=1}^{i} n_{j}$ and $M_{k}=\sum_{j=1}^{k} m_{j}$. Then define

$$
\sigma_{\mu}=\left(\begin{array}{l}
\cdots N_{i}-\left(n_{i}-1\right) \cdots N_{i}-j \cdots N_{i} \cdots \\
\cdots M_{\mu(i)}-\left(n_{i}-1\right) \cdots M_{\mu(i)}-j \cdots M_{\mu(i)} \cdots
\end{array}\right)
$$

Then for $1 \leq j<n_{i}=m_{\mu}(i)$,

$$
\begin{aligned}
& z_{\sigma_{\mu}\left(N_{i}-j+1\right)}-z_{\sigma_{\mu}\left(N_{i}-j\right)}+\frac{1}{2} \\
= & z_{M_{\mu(i)}-j+1}-z_{M_{\mu(i)}-j}+\frac{1}{2} .
\end{aligned}
$$

This means that $z \rightarrow z^{\sigma_{\mu}}$ takes $\mathscr{H}_{n_{1}, \cdots, n_{r}}$ one-to-one, onto $\mathscr{H}_{m_{1}}, \cdots, m_{r}$.

Suppose next that $\zeta_{n_{1}}, \cdots, n_{r} \sim \zeta_{m_{1}}, \cdots, m_{r}$ under $\sigma$. Then $z \rightarrow z^{\sigma}$ takes $\mathscr{H}_{n_{1}, \cdots, n_{r}}$ one-to-one, onto $\mathscr{H}_{m_{1}, \cdots, m_{r}}$. The first claim is that there exists a permutation $\mu$ of $\{1,2, \cdots, \gamma\}$ such that if

$$
N_{\imath}=\sum_{j=1}^{i} n_{j}, \quad M_{i}=\sum_{j=1}^{i} m_{j}
$$

then $\sigma\left(N_{i}\right)=M_{\mu(i)}$.

To see this, note that

$$
\left(z_{N_{i}}-z_{N_{i}-1}+\frac{1}{2}=0\right) \in \mathscr{H}_{n_{1}, \cdots, n_{r}} \text {, but }\left(z_{N_{i}+1}-z_{N_{i}}+\frac{1}{2}=0\right) \notin \mathscr{H}_{n_{1}, \cdots, n_{r}} .
$$

And for all $k \in\{1,2, \cdots, n\}-\left\{N_{1}, N_{2}, \cdots, N_{r}\right\}$,

$$
\left(z_{k}-z_{k-1}+\frac{1}{2}=0\right) \in \mathscr{H}_{n_{1}, \cdots, n_{\gamma}} \text {, and }\left(z_{k+1}-z_{k}+\frac{1}{2}=0\right) \in \mathscr{H}_{n_{1}, \cdots, n_{r}} .
$$

Thus for $\sigma$ to send $\mathscr{H}_{n_{1}, \cdots, n_{r}}$ one-to-one, onto $\mathscr{H}_{m_{1}, \cdots, m_{r}}$, it is necessary that $\sigma\left(N_{i}\right)=M_{\mu(i)}$, for some permutation $\mu$ of $\{1,2, \cdots, \gamma\}$.

The next claim is that if $n_{i}>1$, then $m_{\mu(i)}>1$, and $\sigma\left(N_{i}-1\right)=M_{\mu(i)}-1$. If this is not the case, then $\left(0=z_{N_{i}}-z_{N_{i}-1}+\frac{1}{2}\right) \in \mathscr{H}_{n_{1}}, \ldots, n_{r}$ is mapped by 
$\sigma$ onto $\left(0=z_{M_{\mu}(i)}-z_{\sigma\left(N_{\imath}-1\right)}+\frac{1}{2}\right) \notin \mathscr{H}_{m_{1}}, \cdots, m_{\gamma}$. This is a contradiction.

Thus, by induction, we obtain, for $1 \leq j<n_{i}$, that $\sigma\left(N_{i}-j\right)=M_{\mu(i)}-j$, $m_{\mu(i)} \geq n_{i}$. It must be shown that $m_{\mu(i)}=n_{i}$. If this were not so, then $n_{i}<m_{\mu(i)}$ and $0=z_{M_{\mu(i)}-\left(n_{i}-1\right)}-z_{M_{\mu(i)}-n_{i}}+\frac{1}{2} \in \mathscr{H}_{m_{1}, \cdots, m_{\gamma}}$. Let $M_{\mu(i)}-n_{i}=\sigma(x)$. We know that $M_{\mu(i)}-\left(n_{i}-1\right)=\sigma\left(N_{i}-\left(n_{i}-1\right)\right)$. Now where can $x$ lie in the interval between 1 and $n$ ? It cannot lie between $N_{k-1}$ and $N_{k}$ for any $k$. If it did, then $\sigma(x)$ would be between $M_{\mu(k)}$ and $M_{\mu(k)}-m_{\mu(k)}$. This means $x$ cannot exist, a contradiction.

Note that Theorem 1 implies that " $\sim$ " is an equivalence relation.

Now define $\left[n_{1}, \cdots, n_{r}\right]$ to be the equivalence class of all $\zeta_{m_{1}, \cdots, m_{r}}$ which are related to $\zeta_{n_{1}}, \cdots, n_{\gamma}$ by $\sim$.

Define $C\left(n_{1}, \cdots, n_{r}\right)$ to be the number of elements of $\left[n_{1}, \cdots, n_{r}\right]$. And define $N(n, \gamma)$ to be the number of distinct classes $\left[n_{1}, \cdots, n_{\gamma}\right]$ for fixed $n$ and $\gamma$.

CoRollary. $N(n, \gamma)$ is the number of partitions of $n$ into $\gamma$ parts (disregarding order).

Thus $N(n, \gamma)$ is also equal to the number of partitions of $n$ having largest summand $r$.

Now define $E\left(n_{1}, \cdots, n_{\gamma}\right)$ as the number of functional equations of $\zeta_{n_{1}}, \cdots, n_{\gamma}$ induced by functional equations $\zeta_{(n)}(\rho) \sim \zeta_{(n)}\left(\rho^{\sigma}\right)$, for permutations $\sigma$ of $\{1,2, \cdots, n\}$.

And lastly define $G(n, \gamma)$ to be the number of functions $\zeta_{n_{1}}, \cdots, n_{\gamma}$ for fixed $n$ and $\gamma$. Clearly $G(n, \gamma)=\left(\begin{array}{l}n-1 \\ \gamma-1\end{array}\right)$.

Given $\left(n_{1}, \cdots, n_{r}\right)$, let $\tau$ permute the indices $\{1,2, \cdots, \gamma\}$ so that

$$
\begin{gathered}
n_{\tau(1)}=n_{\tau(2)}=\cdots=n_{\tau\left(r_{1}\right)}<n_{\tau\left(r_{1}+1\right)}=n_{\tau\left(r_{1}+2\right)}=\cdots=n_{\tau\left(r_{1}+r_{2}\right)}<\cdots \\
\left.\left.\cdots=n_{\tau\left(r_{1}+r_{2}+\cdots+r_{s-1}\right.}\right)<n_{\tau\left(r_{1}+\cdots+r_{s-1}+1\right.}\right)=\cdots \\
\cdots=n_{\tau\left(r_{1}+\cdots+r_{s}\right)} .
\end{gathered}
$$

That is, using our usual notation for $\left(r_{1}, r_{2}, \cdots, r_{s}\right)$, namely $R_{j}=\sum_{1}^{J} r_{i}$, we have

$$
n_{\tau\left(R_{j}+1\right)}=n_{\tau\left(R_{j}+2\right)}=\cdots=n_{\tau\left(R_{j+1}\right)}<n_{\tau\left(R_{j+1}+1\right)},
$$

for $j=0,1, \cdots, s$. Here $R_{0}=0$.

Theorem 2. $E\left(n_{1}, \cdots, n_{r}\right)=\left(r_{1}\right) !\left(r_{2}\right) ! \cdots\left(r_{s}\right) !$. 
Proof. The problem is to count the number of permutations $\sigma$ of $\{1,2, \cdots, n\}$ such that $z \rightarrow z^{\sigma}$ fixes $\mathscr{H}_{n_{1}, \cdots, n_{r}}$. By the proof of Theorem 1, such a permutation $\sigma$ has the following properties: $\sigma\left(N_{i}\right)=N_{\mu(i)}$, for some permutation $\mu$ of $\{1,2, \cdots, \gamma\}$. And $\sigma\left(N_{i}-j\right)=N_{\mu(i)}-j$, for $1 \leq j<n_{i}$. Finally $n_{\mu(i)}=n_{i}$.

Therefore, if $i=1, \cdots, s$,

$$
\mu\left(\tau\left(R_{i}+j\right)\right) \in\left\{\tau\left(R_{i}+j\right) \mid j=1,2, \cdots, r_{i+1}\right\}, j=1,2, \cdots, r_{i+1} .
$$

There are exactly $\left(r_{1}\right) !\left(r_{2}\right) ! \cdots\left(r_{s}\right) !$ such $\mu$, and therefore the same number of $\sigma$.

CoROLlaRy. $C\left(n_{1}, \cdots, n_{r}\right)=\frac{r !}{E\left(n_{1}, \cdots, n_{r}\right)}$.

\section{Proof. Clear.}

We shall consider some examples:

1. $r=2, n>1$.

$$
\begin{aligned}
& N(n, 2)=\left[\frac{n}{2}\right] \\
& E\left(n_{1}, n_{2}\right)=1+\delta_{n_{1} n_{2}} \\
& G(n, 2)=n-1
\end{aligned}
$$

2. $r=n-1, n>1$.

$$
\begin{aligned}
& N(n, n-1)=1 \\
& E(1, \cdots, 1,2)=(n-1) ! \\
& G(n, n-1)=n-1
\end{aligned}
$$

3. $n=7$

a. $\gamma=5$

$$
\begin{array}{ll}
N(7,5)=2 & \\
E(2,2,1,1,1)=3 ! 2 ! & C(2,2,1,1,1)=10 \\
E(3,1,1,1,1)=4 ! & C(3,1,1,1,1)=5 \\
G(7,5)=15 &
\end{array}
$$

b. $r=4$

$$
\begin{array}{ll}
N(7,4)=3 & \\
E(4,1,1,1)=3 ! & C(4,1,1,1)=4 \\
E(3,2,1,1)=2 ! & C(3,2,1,1)=12 \\
E(2,2,2,1)=3 ! & C(2,2,2,1)=4 \\
G(7,4)=20 &
\end{array}
$$


c. $\gamma=3$

$$
\begin{array}{ll}
N(7,3)=4 & \\
E(5,1,1)=2 & C(5,1,1)=3 \\
E(4,2,1)=1 & C(4,2,1)=3 ! \\
E(3,3,1)=2 & C(3,3,1)=3 \\
E(3,2,2)=2 & C(3,2,2)=3 \\
G(7,3)=15 &
\end{array}
$$

Added in proof (October 18, 1971):

For another way of obtaining the results in this paper, see

Maass, H., Siegel's Modular Forms and Dirichlet Series, Lecture Notes in Mathematics, Vol. 216, New York, Springer Verlag, 1971.

\section{BiBLIOGRAPHY}

[1] Koecher, Max, "Uber Dirichlet-Reihen mit Funktionalgleichung", J. Reine Angew. Math. 192 (1953), 1-23.

[2 ] Langlands, R.P., "Eisenstein Series", Algebraic Groups and Discontinuous Subgroups, Proc. Symp. in Pure Math. IX, Providence, R.I., A.M.S., 1966.

[ 3 ] Maass, H., "Some Remarks on Selberg's Zeta Functions", Proc. Internatl. Conf. on Several Complex Variables, U. of Maryland, College Park, Md., 1970.

[4] Selberg, A., "Harmonic Analysis and Discontinuous Groups in Weakly Symmetric Riemannian Spaces with Applications to Dirichlet Series", J. Indian Math. Soc. (1956), 4787.

[ 5 ] — , "A New Type of Zeta Function Connected with Quadratic Forms", Report of the Institute in Theory of Numbers, U. of Colorado, Boulder, Colo., 1959, 207-210.

[6] — "Discontinuous Groups and Harmonic Analysis", Proc. Internatl. Cong. of Math., Stockholm, 1962, 177-189

[ 7 ] Terras, A., “A Generalization of Epstein's Zeta Function”, Nagoya Math., J. 42 (1971),

University of lllinois 